\title{
Errata
}

\section{Pseudovibrio ascidiaceicola sp. nov., isolated from ascidians (sea squirts)}

Yukiyo Fukunaga, Midori Kurahashi, Kenji Tanaka, Kensuke Yanagi, Akira Yokota and Shigeaki Harayama

International Journal of Systematic and Evolutionary Microbiology (2006), 56, part 2, 343-347

In the description of Pseudovibrio ascidiaceicola sp. nov. on page 347 of the paper, the NBRC accession number for reference strain F10102 of Pseudovibrio ascidiaceicola is incorrectly given as NBRC 10097. The correct accession number for reference strain F10102 is NBRC 100974 (Dr Yasuyoshi Nakagawa, NBRC, personal communication).

Extremely halophilic denitrifying bacteria from hypersaline inland lakes, Halovibrio denitrificans sp. nov. and Halospina denitrificans gen. nov., sp. nov., and evidence that the genus name Halovibrio Fendrich 1989 with the type species Halovibrio variabilis should be associated with DSM 3050

D. Yu. Sorokin, T. P. Tourova, E. A. Galinski, C. Belloch and B. J. Tindall International Journal of Systematic and Evolutionary Microbiology (2006), 56, part 2, 379-388

In the description of Halospina gen. nov. on p. 386, the gender given for the genus name is incorrect. The text should read N.L. fem. n. Halospina. 